\title{
MICROBIOLOGÍA SUBGINGIVAL EN PACIENTES HIV+ CONY SIN TERAPIA ANTI-RETROVIRAL
}

Palabras claves: microbiota subgingival, cultivo microbiológico, terapia anti-retroviral, periodontitis HIV, gingivitis HIV.

\section{RESUMEN}

En cavidad oral, se pueden presentar los primeros signos de infección por HIV, observándose lesiones como leucoplasia vellosa, candidiasis oral, sarcoma de Kaposi, gingivitis y periodontitis ulceronecrosante. El objetivo de este estudio fue el de analizar la microbiota subgingival y el estado periodontal en pacientes HIV $(+)$ con y sin terapia anti-retroviral.

Se incluyeron voluntariamente 22 sujetos con y 9 sin terapia anti-retroviral según lo aprobó el comité de ética en humanos de la Universidad del Valle. Se recolectaron datos clínicos como sangrado al sondaje, profundidad al sondaje, movilidad, supuración e indice de placa. Muestras subgingivales fueron tomadas de los sitios mas profundos para cultivo microbiológico. El análisis estadístico incluyó el coeficiente de correlación de Pearson ( $p<0.05$ ), con un $95 \%$ de rango de confianza y para el análisis descriptivo se empleó el software SPSS versión 10.0 para Windows. Para el análisis de muestras pareadas se aplicó la prueba de Friedman $(p<0.05)$ teniendo en cuenta los datos microbiológicos de los pacientes con terapia y sin terapia anti-retroviral.

\footnotetext{
'Periodoncista, Universidad del Valle.

- Miembro del Grupo de Medicina Periodontal.

${ }^{3}$ Director del Grupo de Medicina Periodontal.
}

\author{
Mónica Escudero \\ Javier Enrique Botero ${ }^{1,2}$ \\ Adolfo Contreras ${ }^{3}$
}

Los resultados mostraron afección periodontal en diferentes grados de severidad y una microbiota subgingival compuesta principalmente por bacilos entéricos gram negativos, Fusobacterium spp y Porphyromonas gingivalis. En el artículo se hace una descripción detallada de los resultados.

Dentro de los alcances de este estudio, la terapia anti-retroviral no afectó la composición de la microbiota subgingival y el estado periodontal. $\mathrm{La}$ microbiota subgingival parece ser similar a la encontrada en pacientes inmunocompetentes con enfermedad periodontal. El papel de los entéricos en la etiopatogenia de la enfermedad periodontal debe ser estudiado más a fondo.

\section{INTRODUCCIÓN}

El HIV (Human Immunodeficiency Virus, por sus siglas en inglés) es un retrovirus que ataca el sistema inmunológico. El virus ataca en especial la población de células $\mathrm{CD} 4$ destruyéndolas y dejando el organismo indefenso. La enfermedad se desarrolla con un periodo asintomático en donde el huésped se convierte en un portador del virus y posteriormente se torna susceptible a infecciones por hongos, bacterias y virus ${ }^{1,2,3}$ Se ha informado que el tratamiento de la infección por HIV con AZT (Zidovudina) como única droga, es inconveniente para combatir las mutaciones y 
el desarrollo de la resistencia del virus. La terapia combinada anti-retroviral y temprana es la tendencia actual. Su objeto es lograr disminuir la carga viral a niveles prácticamente indetectables durante el mayor tiempo posible; asimismo, se intenta lograr un esquema de drogas que resulten más eficaces y menos tóxicas, con la posibilidad de agregar sustancias que regulen el sistema inmunológico, 4

La cavidad oral puede ser el primer sitio donde se manifieste la infección por HIV, de modo que el odontólogo desempeña un papel preponderante en el reconocimiento y diagnostico de estos pacientes. Lesiones como la leucoplasia vellosa, candidiasis oral y sarcoma de Kaposi son las mas comunes en la boca. Otras lesiones incluyen herpes orolabial recurrente y severo, la histoplasmosis, enfermedad periodontal asociada al HIV, gingivitis ulceronecrotizante y xerostomía ${ }^{6} \mathrm{La}$ gingivitis HIV se observa como un eritema lineal que es resistente a la terapia periodontal convencional, petequias difusas extendidas en la mucosa vestibular, sangrado al sondaje, sin formación de bolsas periodontales y niveles de placa bacteriana y calculo variables. Por otra parte la periodontitis HIV se observa como una patologia inflamatoria caracterizada por enrojecimiento, edema, sangrado al sondaje, supuración, destrucción de los tejidos periodontales marginales con exposición de hueso alveolar, formación de bolsas periodontales, movilidad dental y dolor profundo ${ }^{7.8}$. La terapia periodontal convencional consiste en raspaje y alisado radicular y una meticulosa higiene oral ${ }^{9}$. Sin tratamiento, la gingivitis asociada a HIV, puede progresar a estomatitis necrotizante, caracterizada por agudas y masivas ulceraciones destructivas de la encia, que se extienden al paladar y a los tejidos llevando a una exposición ósea ${ }^{10}$.

Un sistema inmune comprometido permite la colonización por microorganismos periodontopáticos como Actinobacillus actinomycetemcomitans, Porphyromonas gingivalis, Bacteroides forsythus y Fusobacterium spp que tienen reconocidos factores de virulencia que pueden ini- ciar la destrucción periodontal ${ }^{4,7}$. Hasta el momento no se han publicado estudios que evalúen la microbiota subgingival y el estado periodontal en pacientes $\mathrm{HIV}+$ con enfermedad periodontal con y sin terapia anti-retroviral. El objetivo del presente estudio fue el de analizar la microbiota subgingival y el estado periodontal en pacientes HIV+ cony sin terapia anti-retroviral.

\section{MATERIALESY MÉTODOS}

Se incluyeron pacientes HIV + con y sin terapia anti-retroviral que desearon participar voluntariamente de los programas de HIV del ISS y Saludcoop en la ciudad de Cali en el periodo Julio de 2001 a Junio de 2002. El estudio fue aprobado previamente por el comité de ética en humanos de la Facultad de Salud de la Universidad del Valle y cada paciente firmó un consentimiento escrito de participación. Solo se incluyeron pacientes que cumplieran los siguientes criterios de inclusión: mayores de 18 años de edad, parcialmente edéntulos, HIV+ con y sinterapia anti-retroviral y diagnostico de HIV confirmado por ELISAy Westernblot.

Se utilizó un formato de historia clínica único que incluyó: anamnesis, periodontograma, índice de placa y fotografias clinicas. Para completar el periodontograma se utilizaron sondas metálicas tipo UNC-15 (Hu-Friedy (e) Corp), todas las medidas fueron realizadas por un solo operador. Con el periodontograma se determinó: (a) el margen gingival (b) surco gingival o bolsa periodontal; distancia desde el margen gingival hasta un punto donde se presenta resistencia, a la penetración de la sonda. Las medidas de nivel de inserción iguales o menores a $3 \mathrm{~mm}$ se consideran como surco gingival y aquellas iguales o superiores a $4 \mathrm{~mm}$ como bolsa periodontal, haciendo aproximación al milimetro más cercano (c) línea mucogingival: distancia desde el margen gingival hasta el límite entre encía queratinizada y la mucosa alveolar. (d) movilidad: cualquier grado de desplazamiento dental en cualquier dirección, estimado con la ayuda de dos instrumentos romos haciendo presión en 
sentido vestíbulo lingual o meso distal; la movilidad se registro así: grado I, movilidad dental de menos de 1 mm en sentido horizontal, grado II, movilidad dental de más de 1 mm en sentido horizontal y grado III cuando existió un desplazamiento dental incrementado de más de $2 \mathrm{~mm}$ tanto en sentido vertical como horizontal ${ }^{11}$.

El sangrado al sondaje y la supuración fueron registrados en el periodontograma, como positiva o negativa de acuerdo a su presencia o no y para cada paciente se calculó el porcentaje de sitios que presentaron sangrado y supuración. Adicionalmente se hizo una descripción de la condición clínica del periodonto teniendo en cuenta: color, forma, textura y consistencia. El índice de placa fue tomado por medio de pastilla reveladora de placa bacteriana, se registran las superficies pigmentadas mesial, distal, vestibular o lingual y se obtiene un porcentaje de superficies con placa bacteriana sobre el numero total de superficies presentes ${ }^{12}$.

Se tomaron muestras microbiológicas subgingivales de la siguiente forma: el sitio de la muestra se aisló con rollos de algodón, se elimino la placa supragingival y se insertaron 3 puntas de papel estériles hasta el fondo de la bolsa periodontal en los sitios mas profundos y se mantuvo por aproximadamente 15 segundos. Las puntas de papel fueron transferidas a un frasco de vidrio con tapa de rosca de $2 \mathrm{ml}$, con VMGA III (Medio de transporte) ( $5 \%$ Bacto-Agar Gelatina, $0.05 \%$ Thiothone E Peptona, $0.2 \%$ Bacto agar, $0.05 \%$ ácido tioglicolico, $0.05 \% \mathrm{~L}$-cisteina- $\mathrm{HCl}, 1.0 \% \mathrm{Na}$ Glycerophosphate, $0.0005 \%$ acetato de fenil-mercurio, 0.0003 azul de metileno, $0.024 \% \mathrm{Ca} \mathrm{Cl} 2$ $6 \mathrm{H} 20,0.042 \% \mathrm{Kcl}, 0.1 \% \mathrm{NaCl}, 0.01 \%$ $\mathrm{MgSO} 4-7 \mathrm{H} 20)^{13}$. Las muestras fueron procesadas en laboratorio de microbiología oral de la Universidad del Valle, máximo las 24 horas siguientes a la toma de la muestra. Los microorganismos fueron mecánicamente dispersados de las puntas de papel con un vortex a máxima velocidad por 45 segundos. La suspensión bacteriana luego fue diluida según el logaritmo base $10 \mathrm{en}$ VMG I $(0.25 \%$ triptosa, $0.25 \%$ thiothone E pep- tona, $0.5 \% \mathrm{NaCl}$ ). Con una varilla de vidrio esterilizada se esparció sobre la superficie del agar $0.1 \mathrm{ml}$ de las disoluciones de $10^{-3}, 10^{-4}$ y $10^{-5} \mathrm{en}$ tres placas con agar-sangre brucellas (BBL Microbiology systems ${ }^{*}$, Cockeysville, $\mathrm{Md}$ ), conteniendo $5 \%$ bacto agar de sangre de cordero defibrinada, $0.2 \%$ eritrocitos de cordero hemolisados, $0.0005 \%$ hemina y $0.00005 \%$ menadiona. Las placas de agar fueron incubadas en los sistemas de cultivo anaerobio (Aaneropack System ${ }^{8}$ : $\mathrm{CO}_{2}$ Oxoid System ${ }^{\varnothing}$ ). Después se incubaron a $35 \mathrm{C}^{\circ}$ por 5 a 7 dias, se determinaron los porcentajes bacterianos en cada placa de agar y se utilizaron los métodos de identificación de Slots ${ }^{14}$ para identificación bacteriana presuntiva. Para la detección de organismos facultativos (A. actinomycetemcomitans) y microorganismos superinfectantes como bacilos entéricos gram negativos se utilizo un medio selectivo TSBV (tripticasa - soya - agar suplementado con 75 ?g/ml de Bacitracina $-5 \mathrm{mg} / \mathrm{ml}$ de Vancomicina y $5 \%$ de suero estéril de caballo). Luego fueron incubados por 3 días en un ambiente con $5 \%$ de $\mathrm{CO}_{2}\left(\mathrm{CO}_{2}\right.$ Oxoid System( $(\mathbb{R})$. Se hizo la identificación de acuerdo a la morfología y pruebas de luz UV, CAAM y MUG de las colonias de A.actinomycetemcomitans, P.gingivalis, Fusobacterium spp, $P$. intermedia / nigrescens, B.forsythus, Eubacterium spp, P.micros, Dialister pneumosintes, Bacilos entéricos negativos y levaduras.

El análisis estadístico incluyo el coeficiente de correlación de Pearson $(p<0.05)$, con un $95 \%$ de rango de confianza y para el análisis descriptivo se empleo el software SPSS versión 10.0 para Windows. Para el análisis de muestras pareadas se aplicó la prueba de Friedman $(p<0.05)$ teniendo en cuenta los datos microbiológicos de los pacientes con terapia y sin terapia anti-retroviral.

\section{RESULTADOS}

Se estudió la microbiota subgingival en un grupo de 31 pacientes HIV $(+)$ distribuidos en dos grupos, uno con terapia anti-retroviral de 22 sujetos 
y otro $\sin$ terapia anti-retroviral de 9 sujetos. Se examinaron 26 hombres y 5 mujeres con un promedio de edad de 37.7 años (rango 24-53). Clinicamente se observó enrojecimiento e inflamación de la encía marginal con una profundidad promedio al sondaje de $4.6 \mathrm{~mm}$ en el grupo con antiretrovirales y $5.1 \mathrm{~mm}$ en el grupo sin anti-retrovirales. El porcentaje de sangrado al sondaje y supuración se presento con un promedio $26.7 \%$ (rango $12.2 \%-70.1 \%$ ) y $0.1 \%$ (rango $0-2 \%$ ) para el grupo con anti-retrovirales y $32.9 \%(11.1 \%$ $75.5 \%)$ y $1.6 \%(0-12.5 \%)$ para el grupo sin antiretrovirales, respectivamente. Se detectó movilidad dental incrementada tipo I con un promedio de $1.6 \%$ en el grupo con anti-retrovirales y $10.4 \%$ en el grupo sin anti-retrovirales (Tablas 1, 2 y 3 ).

Analizando el diagnostico periodontal en el grupo de pacientes HIV $(+)$ con terapia anti-retroviral, $15 / 22(68 \%)$ presentaron periodontitis crónica leve, $3 / 22(13 \%)$ periodontitis crónica moderada, $2 / 22(9 \%)$ gingivitis marginal simple con perdida de inserción pre-existente y $1 / 22(4 \%)$ gingivitis marginal simple (Tabla1). En el grupo de pacientes sin terapia anti-retroviral, $6 / 9$ presentaron periodontitis crónica leve, $2 / 9(22 \%)$ periodontitis crónica severa y $1 / 9(11 \%)$ gingivitis crónica simple (Tabla 2). No se observo ningún paciente en estado de salud periodontal (Foto 1).

El tiempo de diagnostico del HIV promedio fue de 5.9 y 4.1 años para el grupo con anti-retrovirales y sin anti-retrovirales, respectivamente. Adicionalmente, el grupo con terapia anti-retroviral presento un promedio de tiempo de tratamiento de 3.2 años (Rango 1-8 años) y distribuidos en dos tipos de terapia, análogos nucleótidos inhibidores de la transcriptasa inversa (11/22) y análogos nucleótidos inhibidores de la transcriptasa inversa + inhibidores de proteasa (11/22). La tabla 4 muestra los porcentajes de aislamientos de los microorganismos periodontopáticos y superinfectantes subgingivales en pacientes con HIV $(+)$ con y $\sin$ terapia anti-retroviral retrospectivos. Fusobacterium spp fue el microorganismo mas frecuente (17/22) seguido por bacilos entéricos gram negativos $(16 / 22)$ y $P$. gingivalis $(14 / 22) \mathrm{en} \mathrm{el}$ grupo de pacientes con anti-retrovirales. En comparación, los bacilos entéricos gram negativos (7/) 9) fueron los mas frecuentes seguido por $P$. gingivalis (6/9), P. intermedia (6/9) y Fusobacterium spp (6/9). Al analizar la proporción en la microbiota subgingival, $P$. gingivalis presento un $92.9 \%$ y $85.7 \%$, seguido por Fusobacterium spp $88.9 \%$ y $75.3 \%$, bacilos entéricos gram negati$\operatorname{vos} 85.7 \%$ y $71.4 \%$ en el grupo sin anti-retrovirales y con anti-retrovirales, respectivamente.

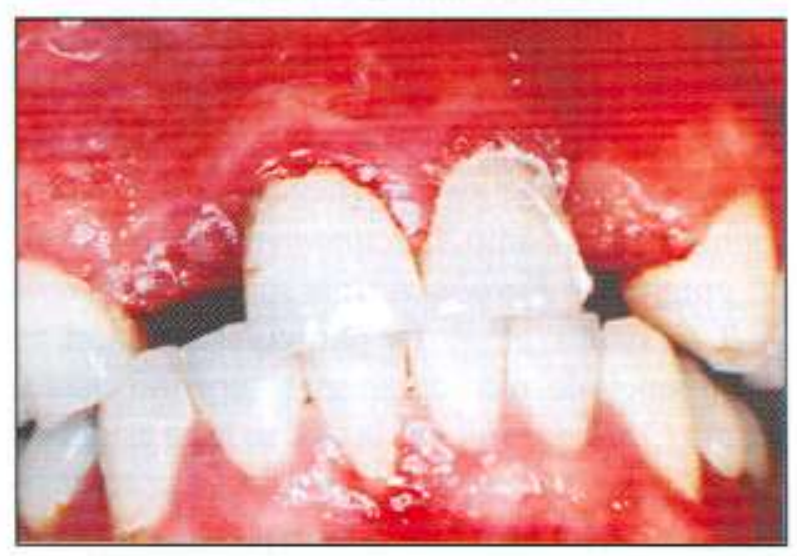

Foto 1. Paciente HIV + con tratamiento anti-retroviral en donde se observa compromiso periodontal severo.

\section{DISCUSIÓN}

En Colombia, se han presentado 172.785 casos de infección por HIV de los cuales $76 \%$ (131.400) son asintomáticos y $24 \%(41.385)$ han desarrollado SIDA desde 1983 hasta 2001. Del total de casos de SIDA, han fallecido $32 \%$ (13.347) de los casos según los registros de defunción del DANE. Si tenemos en cuenta que mediante una encuesta a los Programas territoriales realizada entre septiembre y diciembre del 2001, se identificó que 4044 personas fueron diagnosticadas entre el 2000 y 2001 , y de estos el $58 \%$ iniciaron tratamiento con antiretrovirales ${ }^{15}$. En este estudio se examinaron 22 pacientes HIV positivos bajo terapia antiretroviral con un promedio de duración de 3.2 años y 9 pacientes sin terapia antiretroviral. La meta de la terapia anti-retrovira! 
es la de disminuir el numero de copias de HIVRNA por debajo del nivel de detección. Con tal fin se han diseñado protocolos de manejo que incluyen la combinación de dos o mas antiretrovirales como inhibidores de la transcriptasa reversa e inhibidores de proteasas pero aun así, no se ha podio encontrar una cura para la enfermedad ${ }^{16}$.

En este estudio se analiza los hallazgos clínicos y la microbiota subgingival en pacientes HIV $(+)$ con $y$ sin terapia anti-retroviral. Previamente de ha reportado que la microbiota subgingival en pacientes HIV $(+)$ es muy similar con aquellos pacientes con gingivitis o periodontitis, dependiendo de la severidad del compromiso periodontal. Se observa entonces una microbiota caracterizada por espiroquetas, bacilos gram negativos y los periodontopáticos como A. actinomycetemcomitans. P. gingivalis, Fusobacterium spp, E. corrodens y B. forsythus ${ }^{17,18,19}$. De particular atención, es la presencia de microorganismos superinfectantes como Candida albicans la cual se le ha asignado un rol importante en la progresión del eritema lineal ya que este infiltra el tejido haciendo su control dificil con terapia periodontal convenciona ${ }^{20,21}$. En este estudio solo se encontró un caso $(1 / 22)$ con Candida albicans en el grupo de pacientes con anti-retrovirales detectado por medio de cultivo selectivo. En contraste, la presencia de otros microorganismos superinfectantes como enterobacterias fue mas frecuente en ambos grupos de pacientes con una frecuencia de detección de 16/ 22 y $7 / 9$ en pacientes HIV $(+)$ con anti-retrovirales y sin anti-retrovirales, respectivamente. De forma similar el porcentaje que alcanzaron de la microbiota cultivable subgingival fue alta $(85.7 \%)$. Los bacilos entéricos gram negativos no deben considerarse organismos frecuentes en cavidad oral. También son conocidos como enterobacterias e incluyen todas las especies que causan la diarrea y otras infecciones nosocomiales a nivel de tracto urinario, respiratorio y sistema nervioso central y pueden ser aislados de heces fecales humanas o animales, agua, aguas negras, abono y en casos de infección, sangre y materia fecal ${ }^{23}$. Adicionalmente, se han aislado de soluciones acuosas contaminadas, jabones, esponjas y recientemente de los cepillos dentales ${ }^{22}$ en pacientes con periodontitis. Estos microorganismos son implicados en el $68 \%$ y el $100 \%$ de las infecciones intrahospitalarias y su capacidad de transmisión persona a persona representa un gran problema de salud publica ${ }^{23}$ y muchas de estas infecciones terminan causando sepsis y llevando a la muerte. Los bacilos entéricos gram negativos tienen la capacidad de producir potentes factores de virulencia como adhesinas invasivas, proteínas antifagociticas y enterotoxinas ${ }^{24}$ que al ser liberados al medio subgingival causan un proceso inflamatorio con la resultante de un proceso destructivo crónico de los tejido periodontales.

Hasta el momento no se ha esclarecido el papel que cumplen estos microorganismos superinfectantes en la etiopatogénesis de la enfermedad periodontal. Slots $\mathrm{et}^{2 \mathrm{a}^{25}}$ encontraron una alta prevalencia de mas de $50 \%$ de bacilos entéricos gram negativos con un $23 \%$ de la microbiota cultivable en Republica Dominicana. Los resultados mostraron una mayor frecuencia de bacilos entéricos gram negativos que los microorganismos relacionados con periodontitis. Wahab et $\mathrm{al}^{26}$ encontraron resultados similares en Sudan. Es posible que estos microorganismos tengan un papel importante en la patogénesis de la destrucción de los tejidos periodontales ${ }^{27}$ y mas aun, sabiendo que la bolsa periodontal sirve como reservorio de microorganismos superifectantes que se pueden diseminar a través de la sangre o el tracto grastro-intestinal, tal vez sean importantes en los procesos infecciosos generales en los pacientes HIV $(+)$.

Fusobacterium spp, $P$. gingivalis y $P$. intermedia fueron los microorganismos periodontopáticos mas frecuentes y con los porcentajes de microbiota subgingival cultivable mas altos (Tabla 4). Estudios muestran que la microbiota subgingival en pacientes HIV $(+)$ y HIV $(-)$ con periodontitis no difieren significativamente ${ }^{28}$. Los resultados de este estudios están de acuerdo con tales hallazgos comparando pacientes HIV $(+)$ con y sin terapia anti-retroviral. Sin embargo, cabe recordar que no se incluyo grupo control de pacientes HIV (-) 
pero que si se comparan los datos obtenidos acá con los resultados de estudios anteriores ${ }^{28}$, se puede observar que la composición de la microbiota subgingival es similar.

Los mismos factores de virulencia (colagenasas, factores inhibitorios de la función inmunitaria) producidas por bacterias periodontopáticas en parte explican el proceso destructivo de los tejidos periodontales en estos pacientes ${ }^{29}$. Asi por ejemplo, P.gingivalis, es un patógeno exógeno ${ }^{30}$, que es capaz de infectar los sitios periodontales adhiriéndose a las células epiteliales, los microorganismos o la superficie dental, compitiendo efectivamente con la población microbiana residente y por los mecanismos de defensa humorales y celulares del huésped ${ }^{31}$. P.gingivalis, se presenta en grandes cantidades en los sitios que exhiben inflamación y pobre higiene oral, $\mathrm{y}$ muy poco frecuente en las superficies dentales limpias $^{32}$. Adicionalmente, en estos pacientes se observa una respuesta inmune reducida que puede contribuir a la pobre resistencia del tejido periodontal a bajos niveles de microorganismos periodontopatico ${ }^{33}, 34,35,36$.

Estudios han mostrado que no existen diferencias significativas en la destrucción periodontal entre pacientes HIV $(+)$ con periodontitis y pacientes control $^{37}$. En nuestro caso, se comparó si existía diferencia en la magnitud de la destrucción periodontal en pacientes HIV $(+)$ con y sin terapia antiretroviral. No se encontraron diferencias significativas en la profundidad al sondaje entre ambos grupos, no obstante, fue mayor en el grupo sin terapia anti-retroviral $(5.1 \mathrm{~mm}$ vs $4.6 \mathrm{~mm})$. De forma similar, el sangrado al sondaje fue mayor en ese grupo ( $32.9 \%$ vs $26.7 \%$ ). Debido al diseño transversal del estudio, no es posible establecer explicación que sustenten este fenómeno y se necesitaría una cohorte mucho mas grande con un grupo control seguida por un largo periodo de tiempo que permita sacar conclusiones mas definitivas. Sin embargo, existe una carencia de estudios de enfermedad periodontal en pacientes HIV $(+)$ con y sin terapia anti-retroviral en la literatura disponible y solo se pueden hacer comparaciones con estudios de pacientes con y $\sin$ HIV. Un hallazgo importante de esos estudios es que si es posible encontrar una mayor severidad de la enfermedad periodontal en pacientes HIV $(+)$, al compararlos en un tiempo menor de un año, no se podrían observar diferencias. Los resultados de este estudio están de acuerdo con los hallazgos clinicos encontrados en otros estudios con similares debilidades en diferentes poblaciones ${ }^{7,38,39}$.

Para finalizar, tomando en cuenta el riesgo en que se encuentran estos pacientes de desarrollar infecciones oportunistas que de alguna forma pueden estar relacionadas con focos infecciosos en cavidad oral (bolsa periodontal), seria racional pensar en mantener en ellos una buena salud periodontal para mejorar su condición de vida. Ya se ha demostrado que haciendo mantenimiento periodontal frecuente que incluyan terapia mecánica, control de placa y análisis microbiológico, se pueden mantener los niveles de inserción de forma estable y los microorganismos periodontopáticos y superinfectantes al mínimo $0^{40}$ $\mathrm{y}$ de esta forma reducir la incidencia de enfermedad periodontal en pacientes HIV $(+)$.

\section{CONCLUSIONES}

1. La terapia anti-retroviral parece no afectar la microbiota subgingival en pacientes HIV $(+)$. En ambos grupos de pacientes se observo una composición microbiológica similar.

2. Se encontró una alta detección de bacilos entéricos gram negativos en pacientes HIV $(+)$ con y sin terapia anti-retroviral. El papel de estos microorganismos en el desarrollo de la enfermedad periodontal queda por resolver.

3. Microorganismos periodontopaticos como Fusobacterium spp, $P$. gingivalis y $P$. intermedia fueron detectado con alta frecuencia en pacientes HIV $(+)$ independiente de la terapia anti-retroviral.

4. Existe una similitud entre la microbiota subgingival en pacientes HIV $(+)$ y aquella en- 
contrada en pacientes HIV $(-)$ con enfermedad periodontal.

\section{RECOMENDACIONES}

Se debe aumentar el tamaño de la muestra pues se encontró una tendencia al aumento en la severidad de los parámetros clínicos en pacientes HIV $(+)$ que no reciben terapia anti-retroviral. El entender como es afectada la microbiota subgingival en pacientes HIV+ puede generar un conocimiento nuevo útil en el tratamiento de la enfermedad periodontal en estos pacientes y poder ofrecerles una mejor calidad de vida.

\section{SUMMARY:}

The oral cavity could be the primary place for the establishment of lesions associated with HIV infection including leukoplasia, Kaposi sarcoma, oral candidiasis, ulcerative gingivitis and periodontitis. This study evaluated the subgingival microbiota and periodontal status in HIV $(+)$ patients with and without anti-retroviral therapy.

Twenty two subjects with anti-retroviral therapy and nine subjects without treatment anti-retroviral therapy participated voluntarily in the study, which was approved by the committee on human research of the Universidad del Valle. Clinical data including bleeding on probing, suppuration, pocket probing depth, mobility and plaque index were recorded. Subgingival microbial samples were collected from the deepest sites for culturing analysis. Statistical analysis was carried out using the Pearson correlation test, Friedman test and SSPS software for Windows $(p<0.05)$.

Different states of severity in the periodontal lesions and a subgingival microbiota that encompassed gram negative enteric rods, Fusobacterium spp and Porphyromonas gingivalis were observed. Adetailed description of findings is given in the results of the study.

On considering the limitations of the study, antiretroviral therapy did not influence the subgingival microbiota and periodontal state in HIV $(+)$ patients. The subgingival microbiota appears to be similar to that found in normal patients with periodontal disease. The role of gram negative enteric rods in the etiopathogenesis of periodontal disease requires further research in the future.

Keywords: subgingival microbiota, microbial culture, anti-retroviral therapy, HIV periodontitis, HIV gingivitis.

\section{REFERENCIAS}

1 Carrasquilla G. Epidemiologia de la infección por el virus de la inmunodeficiencia humana en Colombia. Colombia Medica 1996;27:27-32.

2 Itin $\mathrm{PH}$, Lautenschlager S. Viral lesions of the mouth in HIV-infected patients. Dermatol 1997; 194: 1-7.

3 Connolly $L$., Jenkins M. Strategies for anti-retroviral therapy based on the retroviral life cycle. Cohen P.T., Sande M.A., Volberding P.A. The AIDS knowledge base. Second Edition, San Francisco, 1994:3.5, 1-24.

4 Ryder MI. Periodontal considerations in the HIV positive patient. Position paper for the research science and therapy committee. Chicago: American academy of Periodontology 1994.

5 Margiotta V, Campisi G, Mancuso S, Accurso V, Abbadessa V. HIV infection : oral lesions, CD4 cell count and viral load in an Italian study population. JOral Pathol Med $1999 ; 28: 173-177$.

6 Lamster I.B, Grbic J.T., Bucklan R.S., Mitchell-Lewis D., Reynolds H.S., Zambon J.J. Epidemiology and diagnosis of HIV associated periodontal diseases. Oral Diseases 1997;3 suppl 1: S141-S148.

7 Holmstrup P., Westergaard J. Periodontal diseases in HIV patients. J Clin Periodontol 1994:21:270-280.

8 Robinson P.G. Which periodontal changes are associated with HIV infection?. J Clin Periodontol 1998:25:278-285.

9 Gras M. Williams CA. Winkler JR Murray PA. Management of HIV - associated periodontal diseases. In perspectives on oral manifestations of AIDS. Proceedings of the first International AIDS Conference 1988 in San Diego CA. Littleton, MA PSG Publishing 119-130.

10 Gras M. Williams CA. Winkler JR. HIV associated periodontitis complicated by necrotizing stomatitis . Oral Surg Oral Med Oral Pathol 1990, 69-75. 
11 Nyman S. Lindhe J. Exploración de pacientes con enfermedad periodontal. En : Lindhe J. Periodontologia clínica e implantología odontológica. España. Panamericana 2000.387-399.

12 O'Leary TJ, Drake RB, Naylor JE. The plaque control record. J Periodontol 1972;43:38.

13 Moller AJR. Microbiological examination of root canals and periapical tissues of human teeth. Odontol Tidskr 1996;74:1-38.

14 Slots J. Rapid identification of important periodontal microorganisms by cultivation. Oral Microbiol Inmunol 1986:1:48-55.

15 Acosta J, Prieto F, Mejía A. Informe preliminar de respuesta territorial de la situación de las enfermedades de transmisión sexual/HIV/sida Colombia 2001. http:/ /ww w. hiv-lac-epinet.org/media/ ?cat $=62$ \&sub select=1. Visitado el 2 de Marzo de 2003.

16 Phair, J.P. Medical management of HIV infected patients. Periodontology $20002000 ; 23: 78-84$.

17 Rosenstein, D.I., Riviere, GR., Elott, K.S. HIV associated periodontal disease: a new oral spirochete found. J Am Dent Assoc 1993;124:76-80.

18 Moore, L.V., Moore, W.E., Riley, C., Brooks, C.N., Burmeister, J.A., Smibert, R.M. Periodontal microflora of HIV positive subjects with gingivitis or adult periodontitis. J Periodontol 1993;64:48-56.

19 Zambon, J.J., reynolds, H.S., Genco, R.J. Studies of the subgingival microflora in patients with acquired immunodeficiency syndrome. J Periodontol 1990:61:699-704.

20 Gomez, R.S., da Costa, J.E., Loyola, A.M., de Arujo, N.S., de Arujo, V.C. Immunohistochemical study of linear gingival erythema from HIV positive patients. J Periodontal Res 1995;30:355-359.

21 Odden, K., Schenk, K., Hurlen, B. Candidial infection of the gingival in HIV positive infected persons. J Oral Pathol Med 1994;23:178-183.

22 Contreras A, Astudillo M, Daza LH, Garcia LM, Gaviria PA, Parra B, Rosales H, Jaramillo A. Contaminación microbiana de los cepillos dentales en pacientes con enfermedad periodontal. Revista Estomatologia 2002;10:4-14.

23 Abbott S. Klebsiella, Enterobacter: Citrabacter and Serratia en Murray PR (editor). Manual of clinical microbiology, 7 th edition, Washinton D.C. ASM Press, 2001 : paginas 475-482.

24 Aleksic S, Bockemuhl J. Yersinia and other Enterobacteriaceae en Murray PR (editor). Manual of clinical microbiology. 7 th edition, Washinton D.C. ASM Press, 2001 : paginas 483-496.
25 Slots J. Rams T, Feik Det al. Subgingival microflora of advanced periodontitis in the Dominican Republic. J Periodontol 1991;62:543-547.

26 Wahab RA, Vidar Bakken, V, Nilsen R and Nils Skaug. Comparative Detection Frequency of 6 Putative Periodontal Pathogens in Sudanese and Norwegian Adult Periodontitis Patients. J Periodontol 1994;65:1046-1052.

27 Page RC, Kornman KS. The pathogenesis of human periodontitis: an introduction. Periodontol 2000 1997; 14:9-11.

28 Cross, D.L. \& Smith, G.L. Comparison of periodontal disease in HIV seropositive subjects and controls (II) microbiology, immunology and predictors of disease progression. J Clin Periodontol 1995:22:569-577.

29 Haffajee, Socransky SS. Microbial etiological agents of destructive periodontal diseases. Periodontol 2000 1994:5:78-111

30 Van Winkelhoff AJ. Rams TE. Slots J. Systemic antibiotic therapy in periodontics. Periodontology $20001996 ; 10: 45-78$

31 Holt SC. Kesavalu L. Walker S. Genco Ca. virulence factors of Porphyromonas gingivalis. Periodonto$\log y 20001999 ; 20: 168-238$

32 Slots J, Gibbons RJ. Attachment of Bacteroides melaninogenicus subsp. Asaccharolyticus to oral surfaces and its possible role in colonization of the mouth and of periodontal pockets. Infec inmun 1978; $19: 254-264$

33 Gómez, R.S, de Souza, P.E., da Costa, J.E., Araujo, N.S. CD 30+ lymphocytes in chronic gingivitis patients from HIV positive patients: a pilot study. J Periodontol 1997;68:881-883.

34 Steinsvoil, S., Myint, M., Odden, K., Berild, D., Schenk, K. Reduced serum IgG reactivities with bacteria from dental plaque in $\mathrm{HIV}$ infected persons with periodontitis. J Clin Periodontol 1997;24:823-829.

35 Myint, M., Odden, K. Scheurs, O., Halstensen, T.S., Schenk, K. The gingival plasma cell infiltrate in HIV positive patients with periodontitis is disorganized. J Clin Periodontol 1999;26:358-365,

36 Myint, M., Yuan, Z.N., Schenk, K., Reduced numbers of Langherhans cells and increased HLA DR expression in keratinocytes in the oral gingival epithelium of HIV infected patients with periodontitis. $\mathrm{JClin}$ Periodontol 2000;27:513-519. 
37 Robinson, P.G., Boulter, A., Birnbaum, W., Johnson, N.W.A controlled study of relative periodontal attachment loss in people with HIV infection. J Clin Periodontol 2000;27:273-276.

38 Robinson, P.G., Sheiman, A., Challacombe, S.J. \& Zakrzewska, J.M. The periodontal health of homosexual men with HIV infection: a controlled study. Oral Diseases 1996;2:45-52.
39 Teanpaisan, R., Douglas, C.W.1., Nittayananta, W. Isolation and genotyping of black pigmented anaerobes from periodontal sites of HIV positive and non infected subjects in Thailand. J Clin Periodontol 2001;28:311-318.

40 Hofer, D., Hämmerle, G.M., Lang, N.P. Long term results of supportive periodontal therapy (SPT) in HIV seropositive and HIV seronegative patients. J Clin Periodontol 2002;29:630-637.

Correspondencia:

Adolfo Contreras

Escuela de Odontología, Universidad del Valle, Cali, Colombia

Calle $3 \mathrm{~A} \#$ \# 36B-00 San Fernando

adolfoco@yahoo.com

Grupo de investigación:

Grupo de Medicina Periodontal

Facultad de Odontologia

Universidad del Valle

Cali 
Tabla1. Descripción demografica y clinica periodontal de pacientes HIV $(+)$ con terapia antiviral

\begin{tabular}{|c|c|c|c|c|c|c|c|c|c|c|c|c|c|c|}
\hline \multirow[b]{2}{*}{ Sujeto } & \multicolumn{4}{|c|}{ Genero } & \multicolumn{3}{|c|}{ Parametros Clinicos } & \multicolumn{4}{|c|}{ Tiempo } & \multicolumn{3}{|c|}{ Parametros de laboratorio } \\
\hline & Edad & $\mathrm{M}$ & $\mathrm{F}$ & BOP $(\%)$ & Sup (\%) & $\operatorname{Mov}(\%)^{\circ}$ & PPD $(\mathrm{mm})$ & Dx Peridontal & $\mathrm{T1}$ & $\mathrm{T} 2$ & Droga antiviral & Carga viral & $\mathrm{CD} 3$ & $\mathrm{CD}_{4}^{*}$ \\
\hline 1 & 43 & $\mathrm{x}$ & & 12,2 & 0 & 0 & 4,0 & $\overline{\mathrm{PCL}}$ & 8 & 2 & ANITI-IP & 0 & 83 & 20,8 \\
\hline 2 & 35 & $x$ & & 14,5 & 2,0 & 0 & 3,0 & GMS-PIP & 12 & 4 & ANITI-IP & alta & 80,9 & 6.7 \\
\hline 3 & 29 & $x$ & & 12,0 & 0 & 4,0 & 4,0 & PCL & 4 & 3 & ANITI-IP & 0 & 0 & 0 \\
\hline 4 & 39 & $x$ & & 24,7 & 0 & 0 & 5,0 & PCL. & 7 & 4 & ANITI-IP & baja & 7,19 & 139 \\
\hline 5 & 41 & $x$ & & 16,1 & 0 & 0 & 4,0 & $\mathrm{PCL}$ & 3 & 3 & ANITI-IP & 0 & 63,8 & 7,1 \\
\hline 6 & 47 & $x$ & & 13,6 & 0 & 4,5 & 4.0 & PCL. & 3 & 3 & ANITI & alta & $190^{\circ}$ & 696 \\
\hline 7 & 40 & $x$ & & 19.7 & 0 & 0 & 5,0 & $\mathrm{PCL}$ & 11 & 2 & ANITI-IP & 0 & 82 & 14,9 \\
\hline 8 & 40 & $x$ & & 18,0 & 0 & 0 & 3,0 & GMS & 7 & 7 & ANITI - IP & 0 & $661^{\circ}$ & 153 \\
\hline 9 & 36 & $x$ & & 70,1 & 0,6 & 0 & 6,0 & $\mathrm{PCM}$ & 7 & 2 & ANITI - IP & alta & 0 & 425 \\
\hline 10 & 24 & $x$ & & 19,4 & 0 & 0 & 5,0 & $\mathrm{PCL}$ & 1 & 1 & ANITI-IP & alta & 6,27 & 92,12 \\
\hline 11 & 42 & $x$ & & 67,2 & 0 & 0 & 4,0 & $\mathrm{PCL}$ & 11 & 8 & ANITI-IP & 0 & 0 & 0 \\
\hline 12 & 42 & $x$ & & 27,2 & 0 & 0 & 5,0 & $\mathrm{PCL}$ & 6 & 4 & ANITI & alta & 0 & 0 \\
\hline 13 & 36 & $x$ & & 17,7 & 0 & 0 & 5,0 & $\mathrm{PCL}$ & 8 & 3 & ANITI-IP & 0 & 0 & 921 \\
\hline 14 & 36 & & $x$ & 29,0 & 0 & 18,5 & 6,0 & PCM & 8 & 6 & ANITI & alta & 35,4 & 41 \\
\hline 15 & 46 & $x$ & & 24,6 & 0 & 0 & 4,0 & $\mathrm{PCl}$ & 1 & 2 & ANITI & baja & 0 & 54,1 \\
\hline 16 & 38 & $x$ & & 20,3 & 0 & 0 & 5,0 & $\mathrm{PCL}$ & 1 & 1 & ANITI & 0 & 0 & 0 \\
\hline 17 & 38 & $x$ & & 21,4 & 0 & 0 & 6,0 & $\mathrm{PCM}$ & 1 & 1 & ANITI & 0 & 0 & 82 \\
\hline 18 & 35 & & $x$ & 22,2 & 0 & 0 & 3,0 & GMS.PIP & 3 & 2 & ANITI & intermedia & 0 & 90 \\
\hline 19 & 48 & $x$ & & 33,3 & 0 & 0 & 5,0 & $\mathrm{PCL}$ & 5 & 3 & ANITI & 0 & $662^{*}$ & 12 \\
\hline 20 & 29 & $x$ & & 20,7 & 0 & 0 & 6,0 & PCM & 4 & 3 & ANITI & alta & $635^{\circ}$ & 170,000 \\
\hline 21 & 42 & $x$ & & 45,5 & 0 & 4,5 & 5,0 & $\mathrm{PCL}$ & 16 & 5 & ANITI & baja & $299^{\circ}$ & 0 \\
\hline 22 & 39 & $x$ & & 38,5 & 0 & 3,1 & 5,0 & $\mathrm{PCL}$ & 3 & 2 & ANITI & baja & 0 & 150 \\
\hline Total & & 20 & 2 & & & & & $\begin{array}{c}\text { PCL } 15 \\
\text { PCM } 3 \\
\text { GMS-PIP } 2 \\
\text { GMS } 1\end{array}$ & & & $\begin{array}{l}\text { ANITI-IP } 11 \\
\text { ANITI } 11\end{array}$ & $\begin{array}{c}\text { Baja } 4 \\
\text { Intermedia } 1 \\
\text { Alta } 7\end{array}$ & & \\
\hline Promedio & 38,4 & & & 26,7 & 0,1 & 1.6 & 4,6 & & 5,9 & 3,2 & & & & \\
\hline $\begin{array}{c}\text { Desviacion } \\
\text { estandar }\end{array}$ & 5.9 & & & 15,9 & 0.4 & 4,1 & 1,0 & & 4,1 & 1,9 & & & & \\
\hline
\end{tabular}

Edad: años cumplidos

Genero: (M) masculino, (F) temenino.

BOP: sangrado al sondaje

Sup: supuracion

Mov : movilidad grado $1,2,3$. ' solo se observo movilidad grado 1 .

PPD: profundidad al sondaje

Dx periodontal; diagnostico periodontal.

PCL: periodontitis cronica leve

PCM: periodontitis cronica moderada

- $<200$ celulas $/ m m 3$ se considera una valor bajo

GMS-PIP: gingivtis marginal simple con perdida de insercion pre-existente GMS: gingivtis marginal simple

Baja: > de 10,000 copias de RNA-VIH/mI

Intermedia: 10,000-100,000 copias de RNA-VIH/ml

Alta: $>100,000$ copias de ANA-VIH/ml

ANITI: analogos nucleotidos inhibidores de la transcriptasa inversa

IP: inhibidores de proteasa

T1: tiempo de diagnosticada la enfermedad en affos.

T2: tiempo de tratamiento en años. 
Tabla 2. Descripción demografica y clinica periodontal de pacientes HIV $(+)$ sin terapia antiviral

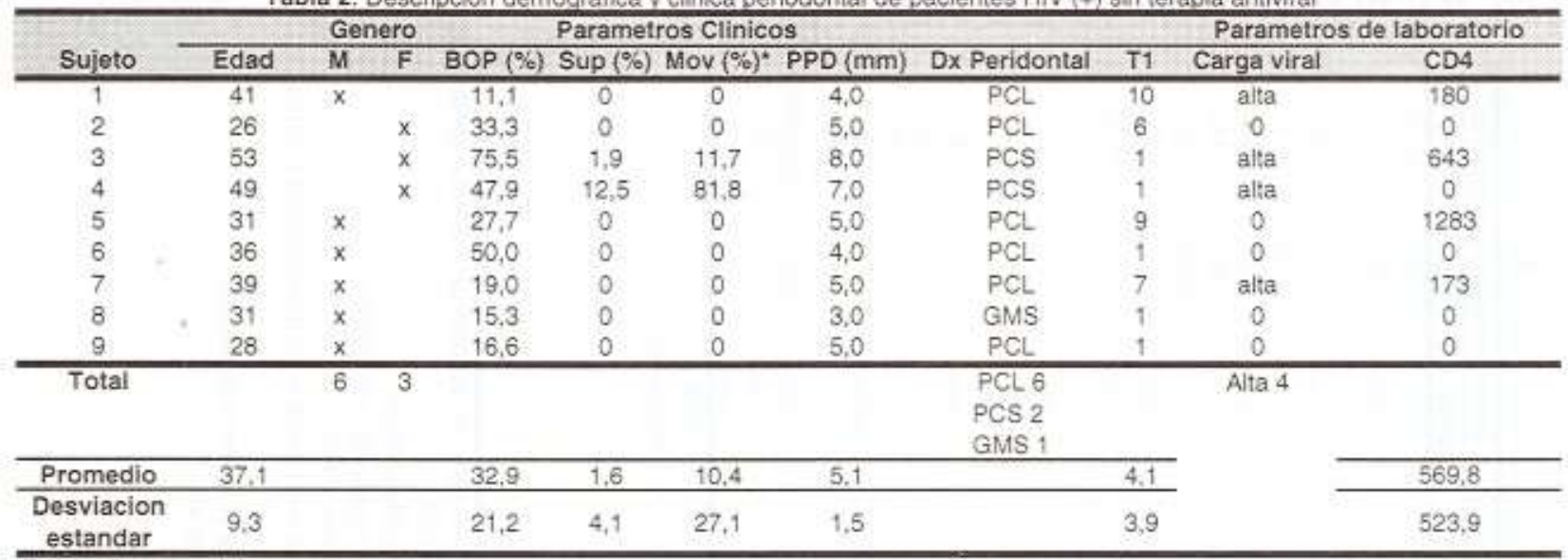

Edad: años cumplidos

Genero: (M) masculino, (F) femenino.

BOP: sangrado al sondaje

Sup: supuracion

Mov: movilidad grado $1,2,3$ * solo se observo movilidad grado 1.

PPD: profundidad al sondaje

Dx periodontal: diagnostico periodontal.

PCL: periodontitis cronica leve

PCS: periodontitis cronica severa

GMS: gingivtis marginal simple

T1: tiempo de diagnosticada la enfermedad en años.

Alta; > 100,000 copias de RNA-VIH/ml

- $<200$ celulas /mm3 se considera una valor bajo

Tabla 3. Comparacion entre los parametros clinicos de los pacientes con y sin terapia antiviral.

\begin{tabular}{ccc}
\hline & \multicolumn{2}{c}{ Pacientes } \\
\hline Parametros clinicos* & Antivirales $(n=22)$ & Sin Antivirales $(n=9)$ \\
\hline BOP $(\%)$ & 26,7 & 32,9 \\
Sup $(\%)$ & 0,1 & 1,6 \\
Mov $(\%)$ & 1,6 & 10,4 \\
PPD $(m m)$ & 4,6 & 5,1 \\
\hline
\end{tabular}

- se expresan los valores promedio.

Tabla 4. Aisiamiento de microorganismos periodontopaticos subgingiveles en pacientes HIV $(+)$ con y sin terapia antiviral.

\begin{tabular}{|c|c|c|c|c|}
\hline \multirow{3}{*}{ Microorganismo } & \multicolumn{4}{|c|}{ Pacientes } \\
\hline & \multicolumn{2}{|c|}{ Antivirales } & \multicolumn{2}{|c|}{ No antivirales } \\
\hline & $\% M S$ & FD & $\%$ MS & FD \\
\hline A. actinomycetemcomitans & 0 & $0 \sqrt{22}$ & 0 & $0 / 9$ \\
\hline P. gingivalis & 85,7 & $14 / 22$ & 92,9 & $6 / 9$ \\
\hline Pintermedia & 38,5 & $=8 / 22$ & 78,6 & $6 / 9$ \\
\hline Biforsythus & 4,6 & $3 / 22$ & 3,6 & $1 / 9$ \\
\hline Campylobacter spp & 14,3 & $=2 / 22$ & 0,25 & $1 / 9$ \\
\hline Eubacterium spp & 24 & ' $5 / 22$ & 0.5 & $1 / 9$ \\
\hline Fusobacterium spp & 75,3 & $17 / 22$ & 88,9 & 69 \\
\hline P.micros & 0 & $0 / 22$ & 0 & $\alpha / 9$ \\
\hline D. pneumosintes & 3.6 & $r \quad 3 / 22$ & 0 & aig \\
\hline Bacilos entericos & 71,4 & $16 / 22$ & 85,7 & $7 / 9$ \\
\hline Estreptococos B hemoliticos & 11 & $=1 / 22$ & 0 & $0 / 9$ \\
\hline Levaduras & 3,4 & $1 / 22$ & 0 & $0 / 9$ \\
\hline
\end{tabular}

$\%$ MS: \% microbiota subgingival

FD: frecuencia de deteccion 